\title{
Модификация целлюлозного сорбента как инструмент регулирования его кинетических характеристик и сорбционной активности по ионам меди (II)
}

\author{
Смирнова Н.Н., Смирнов М.Е. \\ Владимирский государственный университет им. А. Г. и Н. Г. Столетовых, Владимир \\ Поступила в редакцию 28.06.2017 г.
}

\begin{abstract}
В статье приведены результаты сравнительных исследований извлечения ионов меди из водных растворов с использованием немодифицированного и модифицированного целлюлозного сорбента. В качестве модифицирующего агента применяли дитиокарбамат полиэтиленимина Metalsorb FZ. Для повышения устойчивости модификации сорбент дополнительно обрабатывали глутаровым альдегидом. Рассмотрены кинетические и термодинамические особенности сорбционного процесса. Установлено хорошее соответствие экспериментальных результатов и модели псевдовторого порядка. Для математического описания статического равновесия в процессе сорбции были использованы модели Ленгмюра и Фрейндлиха.
\end{abstract}

Ключевые слова: биосорбенты, модификация, ионы тяжелых металлов, сорбция, равновесно-кинетические характеристики.

\section{Cellulose sorbent modification as the regulation instrument of its kinetic characteristics and sorption capacity with regard to copper (II) ions}

\author{
Smirnova N.N., Smirnov M.E. \\ Vladimir State University named after A.G. and N.G. Stoletovs, Vladimir
}

\begin{abstract}
Russia possesses one of the highest water potentials in the world. However, nowadays because of the pollution and foulness the considerable part of rivers and lakes has lost their quality as the drinking water source. In recent years nickel (II), manganese (II), zinc (II) and copper (II) hold the leading position among non-organic contaminants. The essential role in developing water treatment technologies focused on heavymetal ions removal belongs to the sorption methods, including biosorbents which doubtless advantage is the cheap renewable raw material resources base. The main restrictions of biosorbents use are connected with not high kinetic characteristics, low selectivity and comparatively low sorption capacity. The purpose of present investigation was the developing of modification method of cellulose sorbent with enhanced equilibrium and kinetic characteristics and the capability assessment and practicability of its use for $\mathrm{Cu}$ (II) ions extraction from water solutions. Polyethyleneimine dithiocarbamate Metalsorb FZ which can be treated as «integral complexing agent», containing high molecular weight «matrix» (polyethyleneimine) and «active complex former units» (sodium dithiocarbamate), was used as modification agent. To improve the modification process stability the sorbent was additionally cured by glutaric aldehyde. The concentration of dithiocarbamate units in the material was $10 \%(\mathrm{wt})$. The investigations were fulfilled on model systems with $\mathrm{Cu}$ (II) ion's concentrations $0.01-10000 \mathrm{mg} / \mathrm{dm}^{3}$. The content of $\mathrm{Cu}(\mathrm{II})$ ions in the solution has been controlled spectrophotometrically in the presence of diethyldithiocarbamate and by AAS method. The kinetic and thermodynamic characteristics of the sorption process have been observed. For the mathematical description of static equilibrium Langmuir and Freundlich models have been used. It has been found out that the sorption process can be sufficiently described by the kinetic model of pseudo-second order. It has been shown that proposed modification method considerably improves its equilibrium and kinetic characteristics: to enhance
\end{abstract}


the sorption capacity and to cut time of achieving the sorption equilibrium. The possibility of achieving the degree of extraction $90-97 \%$ proclaims the practicability of modified sorbent use in water treatment process for copper ions (II) removal from water solutions in the concentration range not above $1 \mathrm{mg} / \mathrm{dm}^{3}$.

Keywords: biosorbents, modification, heavy-metal ions, sorption, equilibrium and kinetic characteristics

\section{Введение}

Россия обладает одним из самых высоких водных потенциалов в мире. Однако в настоящее время из-за загрязнения или засорения значительная часть рек и озер утратили свои качества как источника питьевого водоснабжения. По экспертным оценкам в 2015 году общий объем загрязненных сточных вод, сброшенных в природные водные объекты, составил 14.4 км$^{3}$ [1]. Лидирующие позиции среди неорганических загрязнителей на протяжении последних лет удерживают никель (II), марганец (II), цинк (II) и медь (II). Существенная роль в разрабатываемых водоочистных технологиях, направленных на удаление ионов тяжелых металлов, отводится сорбционным методам, в том числе с применением биосорбентов [2-8], несомненным преимуществом которых является дешевая возобновляемая сырьевая база. Основные ограничения в использовании биосорбентов связаны с невысокими кинетическими характеристиками, низкой селективностью и сравнительно малой сорбционной емкостью. Решить эти проблемы позволяет их модификация, одним из направлений которой является иммобилизации на поверхности сорбционно-активных комплексообразующих центров [9-12]. Известно, что эффективными комплексообразователями для ионов $\mathrm{Cu}$ (II) являются серо- или азотсодержащие органические соединения, в том числе высокомолекулярные [13].

Разработка способа модификации целлюлозного сорбента с улучшенными равновесно-кинетическими характеристиками и оценка возможности и целесообразности его применения для извлечения ионов $\mathrm{Cu}$ (II) из водных растворов составляла цель настоящего исследования. В качестве модифицирующего агента был использован дитиокарбамат полиэтиленимина Metalsorb FZ, который можно рассматривать как «интегральный комплексообразователь», включающий высокомолекулярную «матрицу» - полиэтиленимин и «активные комплексообразующие фрагменты» - дитиокарбамат натрия.

\section{Эксперимент}

В работе использовали хлопковую целлюлозу (ГОСТ 595-79). Ее модификация включала обработку раствором дитиокарбамата полиэтиленимина (Metalsorb FZ, SNF FLOERGER SA) с концентрацией $0.5 \%$ при $\mathrm{pH}=5$ в течение 10 мин, а затем $0.1 \%$ раствором глутарового альдегида (PANREAC). Отжатый материал высушивали 2 часа в сушильном шкафу при $80^{\circ} \mathrm{C}$. Отмытый в воде и повторно высушенный сорбент использовали при дальнейших исследованиях. Концентрация дитиокарбаматных фрагментов в материале составляла около 10мас.\%. Для ее определения проводили элементный анализ образцов по натрию: навеску сорбента массой 3.0 г сжигали в муфельной печи при температуре $800^{\circ} \mathrm{C}$, а образующийся сухой остаток растворяли в бидистиллированной воде. Концентрацию ионов натрия в растворе измеряли с помощью атомно-абсорбционного спектрометра «КВАНТ - Z.ЭТА».

Спектры пропускания воздушно-сухих образцов снимали на ИКспектрометре ФСМ 1202 с Фурье преобразованием. Растворы с концентрациями ионов меди (II) 0.01 - 100 мг/дм готовили на основе ГСО 7268-96 «Стандартный обра- 
зец состава раствора ионов меди» и бидистиллированной воды. Для приготовления растворов с концентрациями 1-10 г/дм ${ }^{3}$ использовали хлорид меди (II).

Сорбционное равновесие изучали при температуре $25^{\circ} \mathrm{C}$ в статических условиях методом переменных концентраций. Для этого навеску воздушно-сухого сорбента массой 0.1 г заливали $10 \mathrm{~cm}^{3}$ раствора, содержащего ионы $\mathrm{Cu}$ (II) соответствующей концентрации. Эксперимент проводили в течение 6 ч при периодическом перемешивании. Для определения концентрации ионов меди (II) применяли спектрофотометрический (спектрофотометр СФ-2000), основанный на способности ионов меди образовывать окрашенное в желто-коричневый цвет соединение с диэтилдитиокарбаматом натрия и атомно-абсорбционный (атомно-абсорбционный спектрометр КВАНТ - Z.ЭТА) методы.

Сорбционную емкость образцов рассчитывали по формуле:

$$
S=\frac{\left(c_{0}-c\right)}{m} \cdot 0.01
$$

где $S$ - сорбционная емкость образца, мг/г; $c_{0}$ и $c$ - начальная и равновесная концентрации ионов меди в растворе соответственно, мг/дм ${ }^{3} ; m$ - масса сорбента, г.

Количество параллельных опытов составляло не меньше пяти. Результаты, описывающие экспериментальные данные, обрабатывали по теории ошибок. Доверительный интервал рассчитывали для уровня значимости 0.05. Для проведения сорбционного эксперимента в динамических условиях использовали колонку сечением $0.8 \mathrm{~cm}^{2}$. Скорость фильтрации $6 \mathrm{~cm}^{3} /$ мин. Время проведения кинетических исследований составляло 120 минут. Эксперимент проводили при постоянном перемешивании.

\section{Обсуждение результатов}

В литературе представлены разные точки зрения относительно механизма сорбции ионов тяжелых металлов целлюлозными материалами. Однако большинство исследователей склонны рассматривать сорбцию на целлюлозных сорбентах с позиций ионного обмена, предполагая, что катионы взаимодействуют преимущественно с карбоксильными группами целлюлозы $[14,15]$. Тем не менее, в ряде работ показано, что, наряду с ионным обменом, возможно комплексообразование с участием карбоксильных, гидроксильных и карбонильных групп сорбента [16], а также физическая адсорбция [17].

Рассматриваемый в настоящей работе в качестве модифицирующего агента Metalsorb FZ включает два активных компонента, способных к взаимодействию с ионами меди (II): дитиокарбамат и полиэтиленимин. Известно, что взаимодействие $\mathrm{Cu}$ (II) с дитиокарбаматами происходит по донорно-акцепторному механизму, при этом медь образует комплекс с четырехчленными циклами [18]:

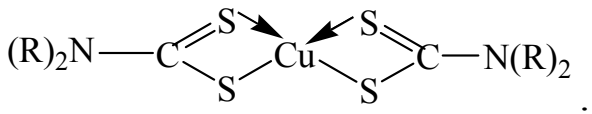

Полиэтиленимин (ПЭИ), в свою очередь, сочетает в Metalsorb функции «матрицы» и комплексообразователя. Было показано, что ПЭИ в зависимости от разветвленности макромолекулярной цепи может связывать ионы меди в соотношении $[\Pi Э И] /[\mathrm{Cu}(\mathrm{II})]$ от 4:1 до 6:1. Причем из-за стерических затруднений координационное число меди в комплексе ПЭИ-Сu (II) равное 4 является предпочтительным [19]: 


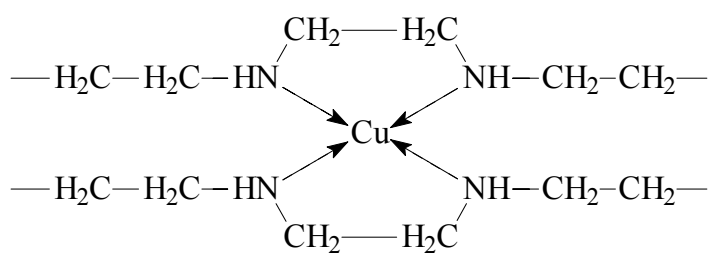

Необходимо учитывать, что ПЭИ, являясь слабым основанием, способен к протонированию и взаимодействию с карбоксильными группами с образованием интерполимерных комплексов, что, в свою очередь, облегчает иммобилизацию Metalsorb на целлюлозной матрице. Установлено, что $\mathrm{pH}=9.8$ соответствует началу протонирования функциональных групп ПЭИ, а при $\mathrm{pH}=4.2$ макромолекулы этого полиэлектролита максимально заряжены [20].

Для повышения устойчивости модификации сорбент может быть дополнительно обработан сшивающим агентом, в частности, глутаровым альдегидом. Результаты, представленные в целом ряде исследований, указывают, что присоединение глутарового альдегида к аминосодержащим соединениям происходит, главным образом, по аминогруппам с образованием межмолекулярных ковалентных связей [21]:
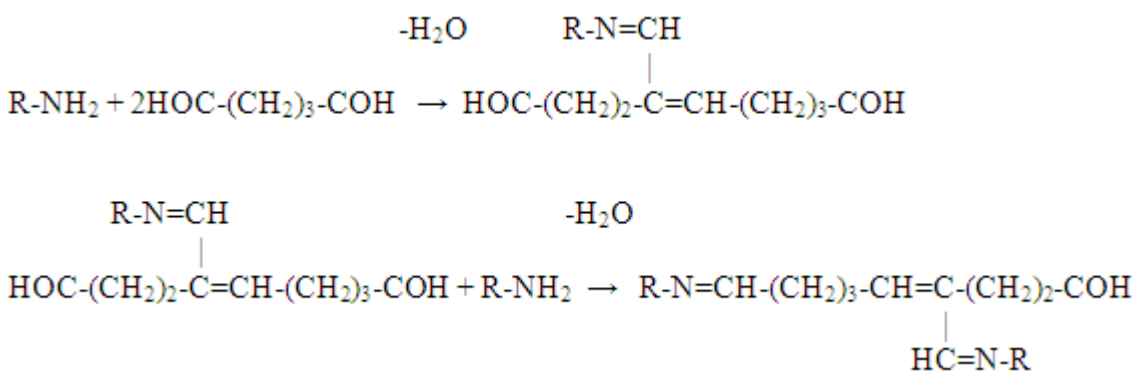

ИК-спектры модифицированного и немодифицированного материала представлены на рис. 1. Значительные изменения в спектрах сорбентов до и после модифицирования наблюдаются в двух областях: от 700 до $800 \mathrm{~cm}^{-1}$, где фиксируется рост интенсивности поглощения, обусловленный увеличением концентрации метиленовых групп в модифицированном сорбенте, и при 1643 и $1655 \mathrm{~cm}^{-1}$, где возрастание интенсивности в модифицированном образце указывает на увеличение концентрации карбонильных групп и проявляются валентные колебания азометиновой группы.

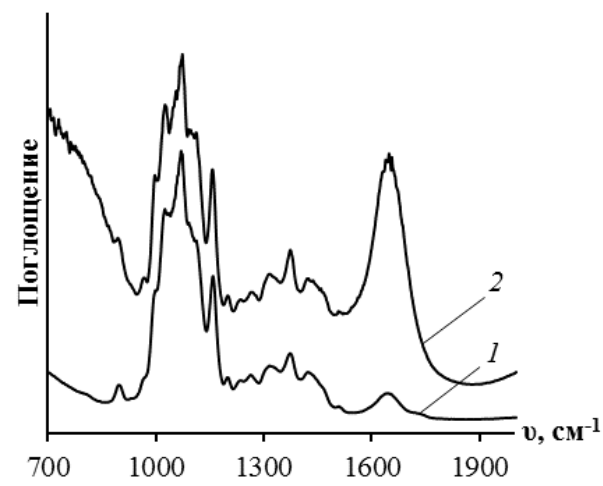

Рис. 1. ИК-спектры немодифицированного (1) и модифицированного Metalsorb (2) образцов хлопковой целлюлозы

Изотермы сорбции $\mathrm{Cu}$ (II) немодифицированным и модифицированным образцами представлены на рис. 2. Для математического описания статического равновесия, устанавливающегося в процессе сорбции, были использованы модели Ленгмюра и Фрейндлиха, наиболее часто применяемые для аналитического описания 
ионообменных равновесий [22-25] . При обработке экспериментальных данных использовали следующие уравнения [26]:

$$
\frac{c}{S}=\frac{1}{b \cdot Q}+\frac{c}{Q}
$$

где $c$ - равновесная концентрация ионов в растворе, мг/дм ${ }^{3} ; S$ - равновесная сорбция, мг/г; $Q, b$ - коэффициенты уравнения Ленгмюра.

$$
\lg S=\lg P+\frac{1}{n} \lg c,
$$

где $P, 1 / n$ - коэффициенты уравнения Фрейндлиха.
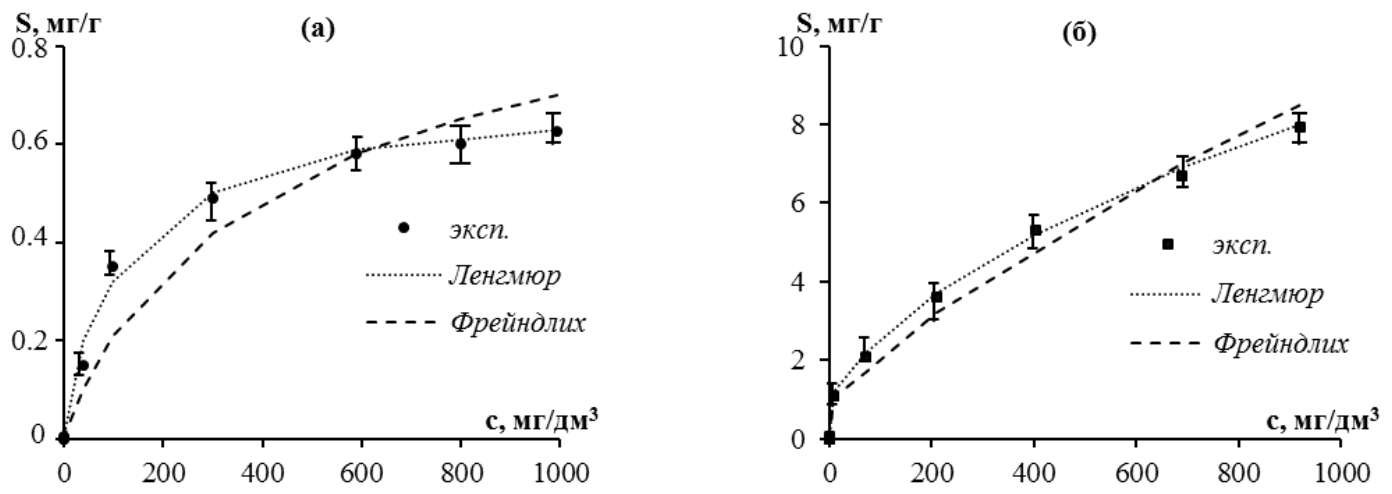

Рис. 2. Экспериментальные и теоретические изотермы сорбции Сu (II) на немодифицированном (а) и модифицированном (б) образцах хлопковой целлюлозы

В табл. 1 приведены значения коэффициентов изотерм сорбции $\mathrm{Cu}$ (II), соответствующие рассматриваемыми математическими моделями. Наблюдаемый при модификации сорбента рост значений Q с 0.894 до 17.882 мг/Г свидетельствует о существенном повышении сорбционной емкости целлюлозного материала.

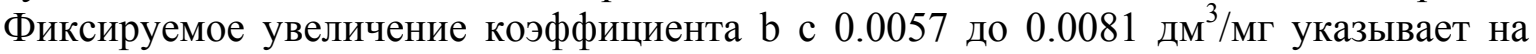
рост сродства в системе сорбент-комплексообразователь/сорбат. Для всех проведенных экспериментов значения коэффициентов $\mathrm{n}$ находятся в диапазоне от 1.39 до 1.63, что свидетельствует о достаточно высокой степени однородности сорбционных центров.

\begin{tabular}{|c|c|c|c|c|c|c|}
\hline \multirow{2}{*}{ Сорбент } & \multicolumn{3}{|c|}{ Модель Ленгмюра } & \multicolumn{3}{|c|}{ Модель Фрейндлиха } \\
\hline & $\mathrm{Q}, \mathrm{Mг} / \Gamma$ & $\mathrm{b}$, дм ${ }^{3} / \mathrm{M \Gamma}$ & $\mathrm{R}^{2}$ & $1 / \mathrm{n}$ & $\mathrm{P}$ & $\mathrm{R}^{2}$ \\
\hline $\begin{array}{c}\text { Немодифицированная } \\
\text { хлопковая целлюлоза }\end{array}$ & 0.894 & 0.0057 & 0.998 & 0.716 & 0.005 & 0.936 \\
\hline $\begin{array}{c}\text { Хлопковая целлюлоза } \\
\text { модифицированная } \\
\text { Metalsorb }\end{array}$ & 17.882 & 0.0081 & 0.998 & 0.614 & 0.273 & 0.967 \\
\hline
\end{tabular}

Таблица 1. Коэффициенты изотерм сорбции ионов $\mathrm{Cu}$ (II)

На рис. 3 показаны результаты динамических исследований сорбции $\mathrm{Cu}$ (II) на немодифицированном и модифицированном образцах хлопковой целлюлозы. Кинетические кривые сорбции ионов меди представлены на рис. 4.

Вопрос о лимитирующей стадии процесса сорбции ионов тяжелых металлов по-прежнему остается дискуссионным [27]. Однако большинство исследователей рассматривают кинетические модели псевдопервого и псевдовторого порядков как наиболее корректно описывающие сорбционные процессы с участием биополимерных сорбентов на основе целлюлозы [28-30]. Причем целый ряд 
публикаций указывает на предпочтительное использование модели псевдовторого порядка[29, 30].

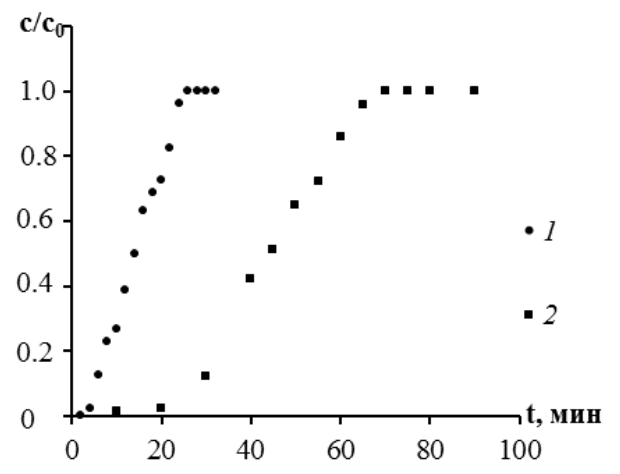

Рис. 3. Динамические выходные кривые сорбции ионов $\mathrm{Cu}$ (II) на немодифицированном (1) и модифицированном (2) образцах хлопковой целлюлозы; $\mathrm{c}_{0}\left(\mathrm{Cu}^{2+}\right)=0.1 \mathrm{мг} /$ дм $^{3}, \mathrm{~T}=23^{\circ} \mathrm{C}$

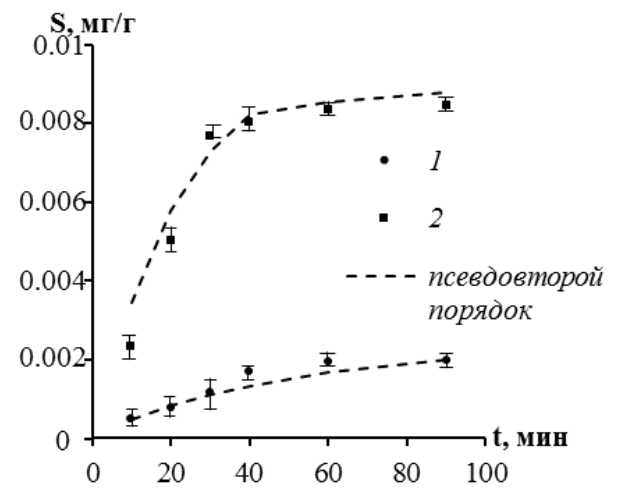

Рис. 4. Экспериментальные и теоретические (модель псевдовторого порядка) кинетические кривые сорбции $\mathrm{Cu}$ (II) на немодифицированном (1) и модифицированном (2) образцах хлопковой целлюлозы

При математической обработке экспериментальных кинетических кривых в настоящей работе использовали следующие уравнения [28, 29]:

$$
\begin{gathered}
\ln \left(S_{e}-S_{t}\right)=\ln S_{e}-k_{1} t \\
\frac{t}{S_{t}}=\frac{1}{k_{2} S_{e}^{2}}+\frac{t}{S_{e}},
\end{gathered}
$$

где $S_{t}$ - сорбционная емкость исследуемого образца за время сорбции $t, \mathrm{Mг} / \Gamma$; $S_{e}$ - равновесная сорбционная емкость исследуемого образца, мг/г; $t$ - время, мин; $k_{l}$, $k_{2}$ - константы скорости согласно модели псевдопервого и псевдовторого порядков, мин $^{-1}, \Gamma^{\cdot} \cdot \mathrm{M}^{-1} \cdot$ мин $^{-1}$.

Представленные в табл. 2 кинетические параметры исследуемого процесса свидетельствуют о значительном улучшении кинетических характеристик целлюлозного сорбента после его модификации. Характер зависимостей, изображенных на рис. 4, указывает на хорошее соответствие полученных результатов и модели псевдовторого порядка, что вполне согласуется с накопленными в литературе данными.

Таблица 2. Кинетические параметры сорбции ионов $\mathrm{Cu}$ (II)

\begin{tabular}{|c|c|c|c|c|}
\hline \multirow{2}{*}{ Сорбент } & \multicolumn{2}{|c|}{ Псевдопервый порядок } & \multicolumn{2}{|c|}{ Псевдовторой порядок } \\
\cline { 2 - 5 } & $\mathrm{k}_{1}, \mathrm{Mин}^{-1}$ & $\mathrm{R}^{2}$ & $\mathrm{k}_{2}, \Gamma^{2} \cdot \mathrm{M \Gamma}^{-1} \cdot \mathrm{Mин}^{-1}$ & $\mathrm{R}^{2}$ \\
\hline $\begin{array}{c}\text { Немодифицированная } \\
\text { хлопковая целлюлоза }\end{array}$ & 0.0351 & 0.901 & 0.0523 & 0.918 \\
\hline $\begin{array}{c}\text { Хлопковая целлюлоза } \\
\text { модифицированная } \\
\text { Меtalsorb }\end{array}$ & 0.0856 & 0.962 & 0.3851 & 0.948 \\
\hline
\end{tabular}

Результаты сравнительного исследования позволяют оценить эффективность применения модифицированного целлюлозного сорбента для извлечения ионов меди (II) из водных растворов. В качестве образцов сравнения в настоящей работе использованы синтетическая ионообменная смола Dowex 50wx8 (Dow Chemical Company) и уголь активированный на каменноугольной основе Silcarbon S 835 (Silcarbon Aktivkohle Gmbh) (рис. 5). Полученные данные свидетельствуют о достаточно высоких 
сорбционных показателях модифицированной целлюлозы и целесообразности ее применения в процессах водоподготовки при относительно небольших (ниже 1 мг/дм ${ }^{3}$ ) начальных концентрациях $\mathrm{Cu}(\mathrm{II})$.

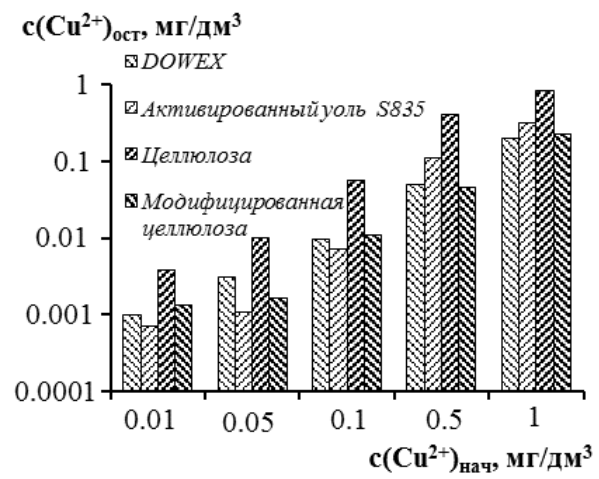

Рис. 5. Влияние природы сорбента на эффективность удаления ионов меди (II) из водных растворов при их различной начальной концентрации

\section{Заключение}

Предложенный способ модификации целлюлозного сорбента позволяет значительно улучшить его равновесно-кинетические характеристики: повысить сорбционную емкость и сократить время достижения сорбционного равновесия. Возможность достижения степени извлечения на уровне 90 - 97\% свидетельствует о целесообразности использования модифицированного сорбента в процессах водоподготовки для удаления ионов меди (II) из водных растворов с концентрацией до 1 мг/дм³

\section{Список литературы}

1. Государственный доклад «О состоянии и охране окружающей среды Российской Федерации в 2015 году». М. Минприроды России. НИА-Природа. 2016. 639 с.

2. Environmental materials and waste. Resource recovery and pollution prevention / Ed. Prasad M.N.V. and Shih Kaimin. London. New York. Acad. Press. 2016, 707 p.

3. Argun M., Dursun S., Ozdemir C., Karatas M. // J. Hazard. Mater. 2007. Vol. 141. pp. 7785.

4. Chakravarty S., Pimple S., Hema S., Chaturvedi T. et al., // J. Hazard. Mater. 2008. Vol. 159. No 2. pp. 396-403.

5. Rao K., Anand S., Venkateswarlu P. // BioResources. 2010. Vol. 5. No 1. pp. 438-454.

6. King P., Srinivasa P., Kumar Y. // J. Hazard. Mater. 2006. Vol. 136. No 3. pp. 560-566.

7. Hubbe M., Hasan S., Ducoste J. // BioResources. 2011. Vol. 6. No 2. pp. 2161-2287.

8. Su P., Granholm K., Pranovich A., Harju L. et al. // BioResources. 2012. Vol. 7. No 2. pp. 2141-2155.

9. Shukla S.R., Pai R.S. // BioResour. Technol. 2005. Vol. 96. pp. 1430-1438.
10. Torres J.D., Faria E.A., Prado A.G.S. // J. Hazard. Mater. B. 2006. Vol. 129.No 1. pp. 239-243.

11. Escudero C., Gabaldon C., Marzal P., Villaescusa I. // J. Hazard. Mater. 2008. Vol. 152. No 2. pp. 476-485.

12. Sato T., Karatsu K., Kitamura H., Ohno Y. // J. Soc. Fiber. Sci. \& Technol. 1983. Vol. 39. No 12. pp. 519-524.

13. Золотов Ю.А., Цизин Г.И., Дмитриенко С.Г., Моросанова Е.И. Сорбционное концентрирование микрокомпонентов из растворов: применение в неорганическом анализе. М. Наука. 2007. 320 с.

14. Юрьев В.И., Позин С.С. // Коллоидный журнал. 1961. Т. 23. № 4. С. 499-503.

15. Никифорова Т.Е., Багровская Н.А., Козлов В.А., Лилин С.А. // Химия растительного сырья. 2009. № 1. С. 5-14.

16. Shukla S.R., Sakhardande V.D. // J. Appl. Polym. Sci. 1992. Vol. 44. № 5. pp. 903-910.

17. Muzarelli R.A.A., Marcotrigiano G., Liu C.-S., Freche A. // Analyt. Chem. 1967. Vol. 39. No 14. pp. 1762-1766. 
18. Подчайнова В.Н., Симонова Л.Н. Медь. М.Наука. 1990. 279 с.

19. Кабанов Н.М., Кокорин А.И., Рогачева В.Б., Зезин А.Б. // Высокомолек. соед. А. 1979. T. 21. № 4. C. 118-126.

20. Бектуров Е.А., Бимендина Л.А., Кудайбергенов С.Е. Полимерные комплексы и катализаторы. Алма-Ата. Наука. 1982. 191 с.

21. Rao P.S., Smitha B., Sridhar S., Krishnoieh A. // Separ. and Purif. Technol. 2006. Vol. 48. No 3. pp. 244-254.

22. Salamatinia B., Kamaruddin A.H., Abdullah A.Z. // J. Appl. Sci. 2007. Vol. 7. No 15. pp. 2020-2027.

23. Sawalha M.F., Peralta-Videa J.R., Romero-Gonzales J., Duarte-Gardea M. et al. // $J$. Chem. Thermodyn. 2007. Vol. 39. No 3. pp. 488-492.

24. Dang V.B.H., Doan H.D., Dang-Vu T., Lohi A. // BioResour. Technol. 2009. Vol. 100. No 1. pp. 211-219.

\section{References}

1. Gosudarstvennyj doklad «O sostoyanii i ohrane okruzhayushchej sredy Rossijskoj Federacii v 2015 godu». Moskva, Minprirody Rossii, NIA-Priroda, 2016, 639 p.

2. Environmental materials and waste. Resource recovery and pollution prevention / Ed. Prasad M.N.V. and Shih Kaimin. London; New York, Acad. Press, 2016, 707 p.

3. Argun M., Dursun S., Ozdemir C., Karatas M., J. Hazard. Mater., 2007, Vol. 141, pp. 7785.

4. Chakravarty S., Pimple S., Hema S., Chaturvedi T. et al., J. Hazard. Mater., 2008, Vol. 159, No 2, pp. 396-403.

5. Rao K., Anand S., Venkateswarlu P., BioResources, 2010, Vol. 5, No 1, pp. 438-454.

6. King P., Srinivasa P., Kumar Y., J. Hazard. Mater., 2006, Vol. 136, No 3, pp. 560566.

7. Hubbe M., Hasan S., Ducoste J., BioResources, 2011, Vol. 6, No 2, pp. 2161-2287.

8. Su P., Granholm K., Pranovich A., Harju L. et al., BioResources, 2012, Vol. 7, No 2, pp. 2141-2155.

9. Shukla S.R., Pai R.S., BioResour. Technol., 2005, Vol. 96, pp. 1430-1438.

10. Torres J.D., Faria E.A., Prado A.G.S., $J$. Hazard. Mater. B, 2006, Vol. 129, No 1, pp. 239-243.
25. Никифорова Т.Е., Козлов В.А. // Журн. Прикл. Химии. 2010. Т. 83. Вып. 10. С. 16421645.

26. Адсорбция из растворов на поверхности твердых тел // Под ред. Парфитта Г., Рочестера К. М. Мир. 1986. 488 с. (Adsorption from solution at the solid/liquid interface / Ed. Parfitt G.D. and Rochester C.H. London, Academic Press, 1983, 416 p.)

27. Никифорова Т.Е., Козлов В.А. // Физикохимия поверхности и защита материалов. 2012. T. 48. № 3. C. 262-266.

28. Zheng L.S., Dang Z., Yi X.Y., Zhang H. // J. Hazard. Mater. 2010. Vol. 176. No 1-3. pp. 650-656.

29. Ghodbane I., Hamdaoui O. // J. Hazard. Mater. 2008. Vol. 160. No 2-3. pp. 301-309.

30. Ghodbane, I., Nouri L., Hamdaoui O., Chilm M. //J. Hazard. Mater. 2007. Vol. 152. No $1 . \quad$ pp. 148-158. 11.Escudero C., Gabaldon C., Marzal P., Villaescusa I., J. Hazard. Mater., 2008, Vol. 152, No 2, pp. 476-485.

12.Sato T., Karatsu K., Kitamura H., Ohno Y., J. Soc. Fiber. Sci. \& Technol., 1983, Vol. 39, No 12, pp. 519-524.

13.Zolotov Yu.A., Cizin G.I., Dmitrienko S.G., Morosanova E.I., Sorbcionnoe koncentrirovanie mikrokomponentov iz rastvorov: primenenie $\mathrm{v}$ neorganicheskom analize, M., Nauka, 2007, $320 \mathrm{p}$.

14. Yur'ev V.I., Pozin S.S., Kolloidnyj zhurnal, 1961, Vol. 23, No 4, pp. 499-503.

15.Nikiforova T.E., Bagrovskaya N.A., Kozlov V.A., Lilin S.A., Himiya rastitel'nogo syr'ya, 2009, No 1, pp. 5-14.

16.Shukla S.R., Sakhardande V.D., J. Appl. Polym. Sci., 1992, Vol. 44, No 5, pp. 903-910.

17.Muzarelli R.A.A., Marcotrigiano G., Liu C.-S., Freche A., Analyt. Chem., 1967, Vol. 39, No 14, pp. 1762-1766.

18.Podchajnova V.N., Simonova L.N. Med'. M., Nauka Publ., 1990, 279 p.

19.Kabanov N.M., Kokorin A.I., Rogacheva V.B., Zezin A.B., Vysokomolek. soed. A, 1979, Vol. 21, No 4, pp. 118-126.

20.Bekturov E.A., Bimendina L.A., Kudajbergenov S.E., Polimernye kompleksy i katalizatory. Alma-Ata, Nauka Publ., 1982, 191 p. 
21.Rao P.S., Smitha B., Sridhar S., Krishnoieh A., Separ. and Purif. Technol., 2006, Vol. 48, No 3, pp. 244-254.

22. Salamatinia B., Kamaruddin A.H., Abdullah A.Z., J. Appl. Sci., 2007. Vol. 7, No 15, pp. 2020-2027.

23.Sawalha M.F., Peralta-Videa J.R., Romero-Gonzales J., Duarte-Gardea M. et al., $J$. Chem. Thermodyn., 2007, Vol. 39, No 3, pp. 488-492.

24.Dang V.B.H., Doan H.D., Dang-Vu T., Lohi A., BioResour. Technol., 2009, Vol. 100, No 1, pp. 211-219.

25.Nikiforova T.E., Kozlov V.A., ZHurn. Prikl. Himii, 2010, Vol. 83, Vyp. 10, pp. 16421645.

Смирнова Наталья Николаевна профессор кафедры химии, д.Х.н., Владимирский государственный университет им. А.Г. и Н.Г. Столетовых, Владимир

Смирнов Максим Евгеньевич - магистрант кафедры химии, Владимирский государственный университет им. А.Г. и Н.Г. Столетовых, Владимир.
26.Adsorption from solution at the solid/liquid interface / Ed. Parfitt G.D. and Rochester C.H. London, Academic Press, 1983, 416 p.

27.Nikiforova T.E., Kozlov V.A. , Fizikohimiya poverhnosti $i$ zashchita materialov, 2012, Vol. 48, No 3, pp. 262-266.

28.Zheng L.S., Dang Z., Yi X.Y., Zhang H., J. Hazard. Mater., 2010, Vol. 176, No 1-3, pp. 650-656.

29. Ghodbane I., Hamdaoui O., J. Hazard. Mater., 2008, Vol. 160, No 2-3, pp. 301-309.

30.Ghodbane, I., Nouri L., Hamdaoui O., Chilm M., J. Hazard. Mater., 2007, Vol. 152, No 1, pp. 148-158.

Smirnova Natalya N. - prof., grand Ph.D (chemistry), department of chemistry, Vladimir State University named after A.G. and N.G. Stoletovs, Vladimir, e-mail: smirnovann@list.ru

Smirnov Maksim E. - undergraduate of the chair of chemistry department, Vladimir State University named after A.G. and N.G. Stoletovs, Vladimir. 